\title{
iência ural \\ ESCANEAMENTO DIGITAL E PROTOTIPAGEM 3D PARA CONFECÇÃO DE LAMINADOS CERÂMICOS: RELATO DE CASO CLÍNICO
}

Digital Scanning and ${ }_{3} D$ Prototyping for Ceramic Laminate Confection: Clinical Case

Luís Felipe Espíndola-Castro | | Universidade de Pernambuco (FOP/UPE) | | Doutorando em Dentística / Endodontia (FOP/UPE) | |

lipe_espindola@hotmail.com

Leonardo Santiago Ortigoza || Universidade de Pernambuco (FOP/UPE) || Mestre em Dentística / Endodontia (FOP/UPE) || leosanorti@gmail.com Gabriela Queiroz de Melo Monteiro || Universidade de Pernambuco (FOP/UPE) | | Doutora em Dentística (FOP/UPE) | | gabriela.queiroz@upe.br

Autor responsável pela correspondência Luís Felipe Espíndola-Castro | | lipe_espindola@hotmail.com 


\section{RESUMO}

Introdução: lentes de contato dentais podem ser uma excelente opção para correção de cor, forma, tamanho e posicionamento dental. Entretanto é um tratamento que requer várias etapas laboratoriais com riscos de distorções nas etapas de moldagem e vazamento do modelo. Desta forma, desadaptações dos laminados em boca podem ser frequentes. Objetivo: relatar um caso clínico de nove laminados cerâmicos em que foi empregado o escaneamento digital, troquelização virtual e prototipagem do modelo em 3D. Método: paciente do gênero feminino, 59 anos, buscou atendimento odontológico queixando-se de desproporcionalidade dentária ao sorrir. Ao exame clínico foi observada uma inclinação maxilar que causava aquela desarmonia. Foi proposta a confecção de laminados cerâmicos para compensar a discrepância óssea bem como melhorar forma, contorno e cromia dentária. Após planejamento digital, confecção do enceramento diagnóstico, mock up, e aceita do planejamento por parte da paciente, iniciaram-se os preparos dentários. Finalizada esta etapa, os dentes foram escaneados (Trios 3Shape), troquelizados e prototipados em impressora 3D. O modelo foi encaminhado ao laboratório que confeccionou as peças protéticas em dissilicato de lítio de forma injetada e maquiada. Por fim, foram realizadas as provas secas, úmidas, ajustes necessários e cimentação dos laminados com cimento resinoso fotopolimerizável. Conclusão: a tecnologia empregada se mostrou eficiente na resolução do caso, sendo uma técnica rápida, que causou pouco desconforto à paciente e oportunizou uma boa adaptação dos laminados cerâmicos.

Palavras-Chave: Estética Dentária; Facetas Dentárias; Cerâmica; Tecnologia
Odontológica

\section{ABSTRACT}

Introduction: laminates veneers can be an excellent choice for color correction, shape, size and dental positioning. However, it is a technique that requires several laboratory steps with risks of distortion in the molding and casting steps of the model. Thus, maladjustments of laminates in the mouth may be frequent. Objective: to report a clinical case of nine ceramic laminates through the digital scanning, virtual punching and 3D prototyping of the model. Methods: A 59 years old woman showed up to dental clinic searched dental care complaining of dental disproportionality when smiling. At the clinical examination, a maxillary inclination was observed that caused disharmony. It was proposed the making of ceramic laminates to compensate the bone discrepancy as well as improve shape, contour and dental color. After the digital planning, preparation diagnostic wax-up, mock up, and acceptance of patient planning, the dental preparations were started. After this step, the teeth were scanned (Trios 3Shape), punched and prototyped in a $3 \mathrm{D}$ printer. The model was sent to the laboratory to do the prosthetic parts in lithium disilicate in an injected and makeup manner. At last, it were made the tests dry, wet, required adjustments finally cementation of the ceramic laminates with photopolymerizable resin cement. Conclusions: the technology employed was efficient lving this case, being a fast technique, which caused little discomfort to the patient rovided a good adaptation of the ceramic laminates.

ords: Esthetics, Dental; Dental Veneers; Ceramics; Technology, Dental.

ista Ciência Plural. 2019; 5(1):113-123

114 


\section{Introdução}

É cada vez mais frequente a busca dos pacientes por um sorriso harmônico. O qual pode ser caracterizado por dentes brancos, grandes, alinhados no arco e proporcionais¹. Para o alcance desta expectativa, a odontologia aliada à tecnologia tem proporcionado cada vez mais procedimentos menos invasivos, previsíveis, eficazes e com alta qualidade estética ${ }^{2,3}$. Nesta perspectiva, lentes de contato dentais podem ser uma excelente opção para correção de cor, forma, tamanho e posicionamento dental ${ }^{4}$. Entretanto, esta é uma técnica que requer várias etapas laboratoriais com riscos de distorções nas etapas de moldagem e vazamento do modelo5,6. Desta forma, desadaptações dos laminados em boca podem ser frequentes ${ }^{5}$.

Para minimizar estas intercorrências, o escaneamento intra oral pode ser útil7. Esse sistema usa uma câmera infravermelha para obter imagens de estruturas bucais e as imagens obtidas são armazenadas e interpretadas por um software, cuja versão 3D cria um modelo tridimensional virtual ${ }^{8}$. O uso desta técnica tem como objetivo simplificar, automatizar e garantir níveis de qualidade com adaptações micrométricas das próteses dentárias 9 . Uma boa definição e acabamento das margens do preparo são essenciais para visualização dos términos cervicais ${ }^{10}$. Entretanto, as moldagens convencionais podem apresentar problemas como a baixa reprodutibilidade das margens dos preparos, o rasgamento de algumas áreas do molde, a presença de debris impregnados no material de moldagem, bolhas e indistinção das margens dos preparos com tecidos moles, após o vazamento do gesso ${ }^{11,12}$.

A partir das imagens obtidas pelo escaneamento intra oral, uma impressora 3D pode transformar as imagens virtuais em modelos prototipados ${ }^{2,13}$. Este técnica permite uma boa reprodução dos tecidos e favorece a troquelização de forma digital e precisa ${ }^{13}$. Deste modo, as chances de alterações interproximais causadas pela serra do troquel diminuem 5,14 . Várias etapas laboratoriais podem ser eliminadas, como: o azamento do gesso nas moldagens, a confecção das réplicas, a colocação dos pinos s modelos além de não necessitar recortar e modelar troqueis $2,15,16$. O registro oclusal hbém é realizado durante o escaneamento, e os modelos são impressos com guia de culação ${ }^{16,17}$. 
Alguns estudos já evidenciam que o escaneamento intra oral apresenta boa capacidade de cópia dos preparos $5,6,12,16,17,18$. Sendo o objetivo do presente artigo, relatar em um caso clínico a confecção de nove laminados cerâmicos em que foi empregado escaneamento intra oral e confecção dos modelos em impressora 3D. Também, objetivou-se avaliar a percepção da paciente quanto a técnica de escaneamento digital, bem como as adaptações em boca das peças protéticas.

\section{Método}

Paciente do gênero feminino, 59 anos, leucoderma, compareceu à consulta odontológica queixando-se de desproporcionalidades dentárias e escurecimento dental. Ao exame clínico inicial, foi observada inclinação maxilar que conferia desproporcionalidades dentárias ao sorrir (Figura 01).

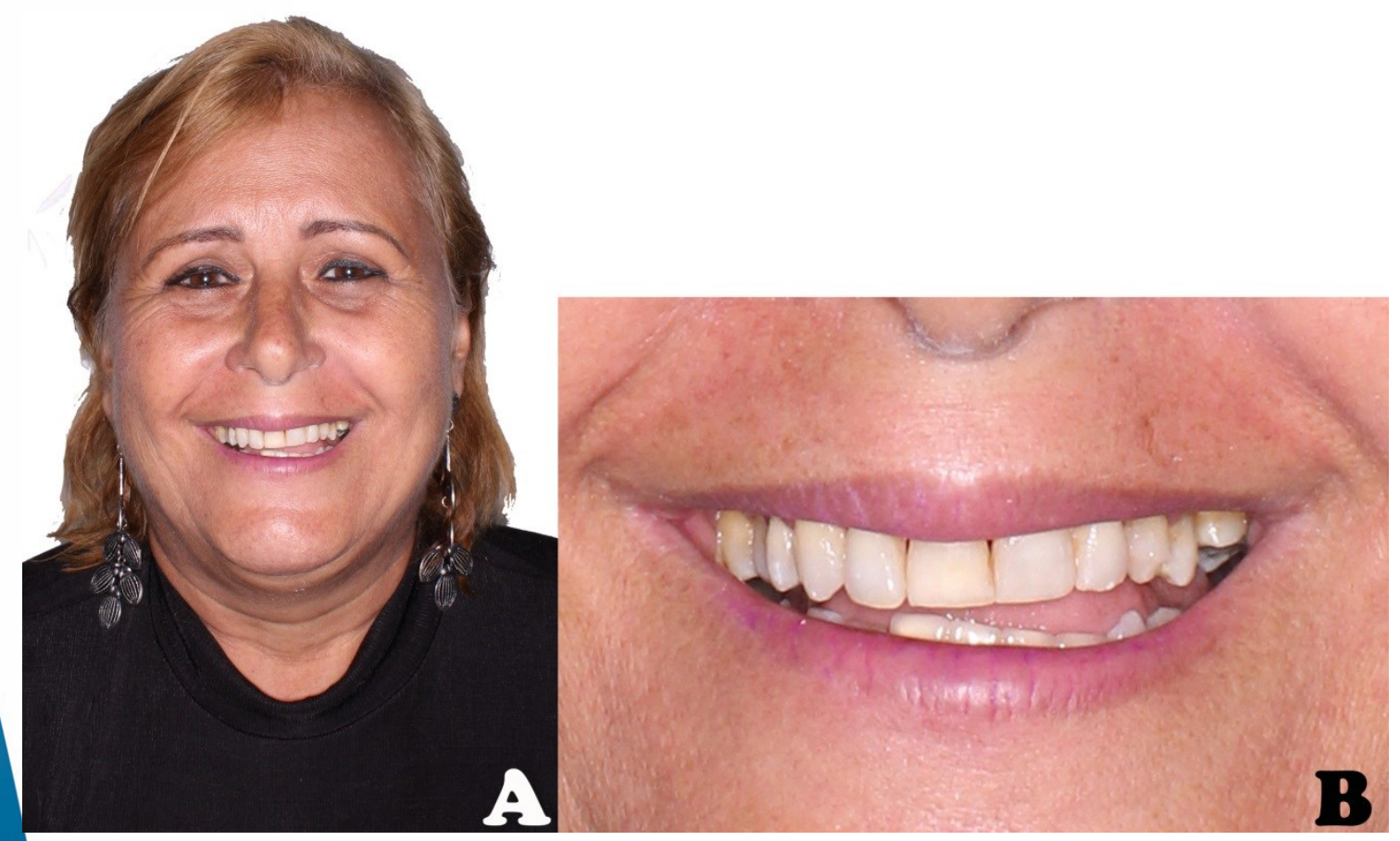

Figura 01: Aspecto clínico inicial. Em A: análise dento facial e em B: vista aproximada do sorriso.

A partir do diagnóstico clínico inicial, foi proposto à paciente a realização de ove laminados cerâmicos para compensar a inclinação maxilar e proporcionar uma hor harmonização do sorriso ajustando forma, contorno e cromia dental. 
Após aceitação do plano de tratamento proposto, foi realizado o planejamento digital do sorriso a partir das fotografias iniciais e confecção de modelos de estudo. Após enceramento diagnóstico baseado no planejamento digital, foi realizado o mock up, e checagem da oclusão, bem como a comunicação com a paciente para avaliar seu nível de expectativa e satisfação com planejamento (Figura 02).

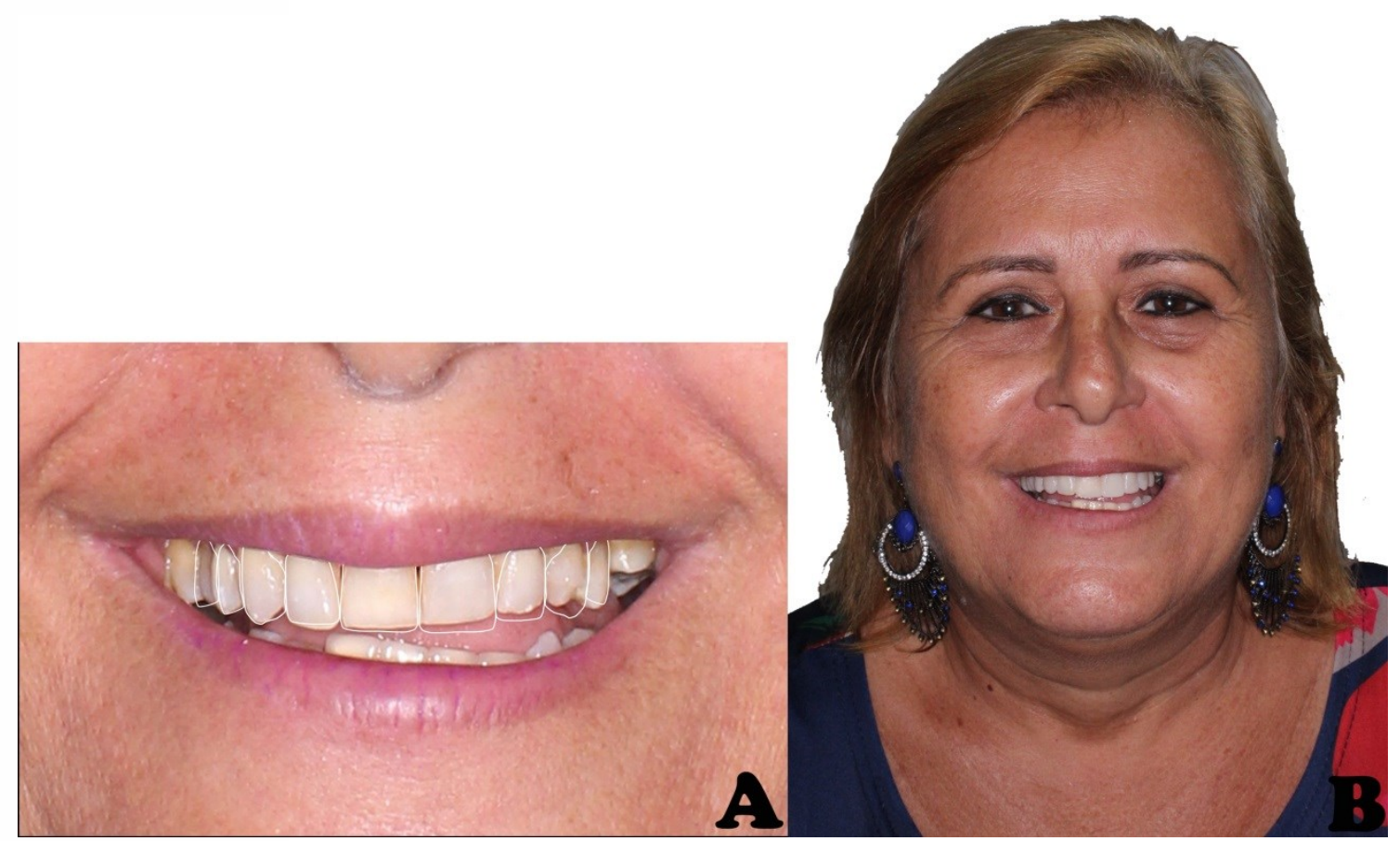

Figura 02: Planejamento do caso clínico. Em A: desenho digital do sorriso e em B: mock up instalado e análise dento facial.

Após aprovação do planejamento, foram realizados os desgastes necessários. A partir do enceramento, foi confeccionado uma guia de desgaste em silicone e executado os preparos dentais. Sequencialmente, foi realizado o escaneamento digital dos arcos dentários gerando assim, imagens virtuais pelo sistema Trios 3Shape (Compenhague, Dinamarca). Com as imagens do escaneamento, foi possível a realização da troquelização de forma digital e posteriormente, impressão do modelo prototipado em 3D (Figura 03). 


\section{ciênncia
purál}
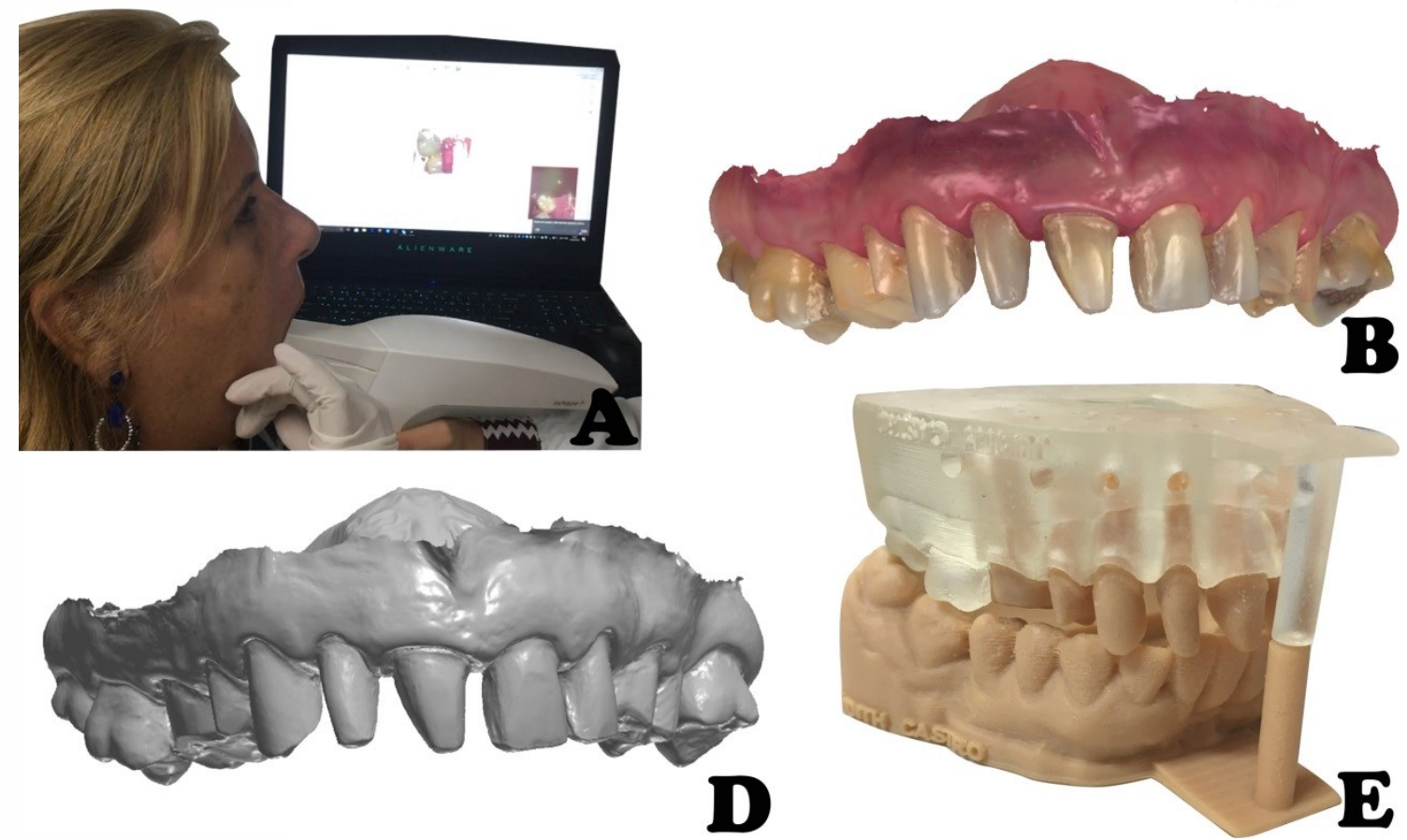

Figura 03: Escaneamento intra oral. Em A: transformação das imagens reais em virtuais. Em B e C: imagens geradas pelo sistema Trios 3 Shape e em D: impressão do modelo troquelizado em 3D em resina com guia de articulação.

O modelo prototipado foi encaminhado ao laboratório para confecção dos laminados cerâmicos em dissilicato de lítio de forma injetada e maquiada. Posteriormente, foi realizada a prova seca, úmida e cimentação dos laminados. As peças foram tratadas em sua face interna com ácido fluorídrico a 10\% (Condac Porcelana / FGM, Joinville, Santa Catarina, Brasil) por 20s, lavagem com água e ar e secagem. Em seguida, aplicação de ácido fosfórico 37\% (Condac / FGM, Joinville, Santa Catarina, Brasil) por 30s, lavagem com spray de água e ar e secagem. Depois, aplicação do silano (Prosil / FGM, Joinville, Santa Catarina, Brasil) aguardando sua evaporação e aplicação do sistema adesivo (Ambar universal / FGM, Joinville, Santa Catarina, Brasil) sem fotopolimerização. A superfície dental foi tratada com ácido fosfórico a $37 \%$ por 30 s em esmalte e 15s em dentina, lavagem com spray de água e ar por igual período, secagem e aplicação do sistema adesivo sem fotopolimerização. Sequencialmente, foi aplicado cimento resinoso fotoativado (Allcem Veneer / FGM, Joinville, Santa Catarina, Brasil) na superfície interna do laminado, levados sobre o ubstrato dental de 2 em 2, remoção dos excessos com pincel a polimerização com fotopolimerizador Bluephase N (Ivoclar Vivadent, Schaan, Liechtenstein). 
Por fim, foi realizado a remoção dos excessos, acabamento e polimento da margem cervical e ajuste oclusal (Figura 04).

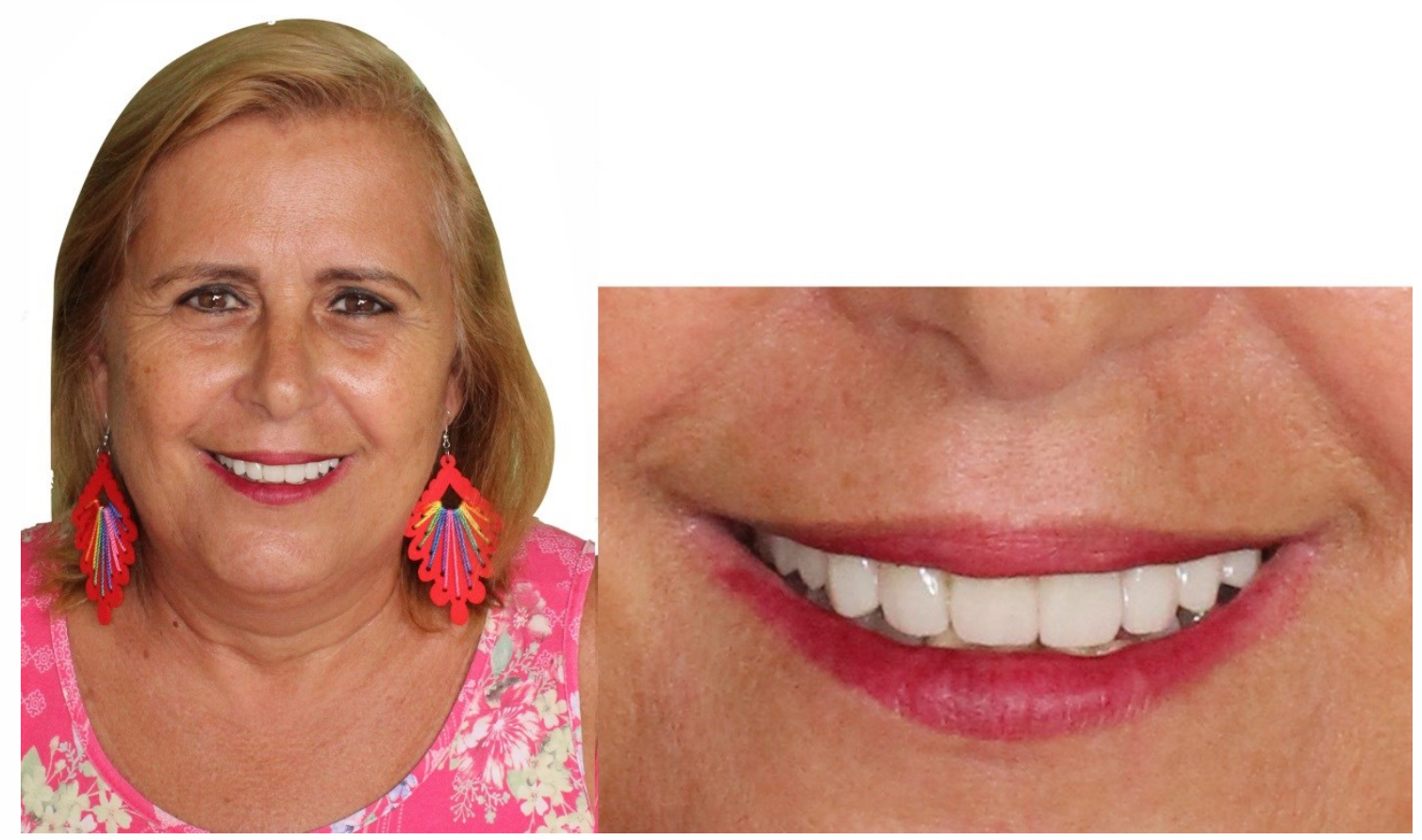

Figura 04: Aspecto clínico final

A paciente autorizou a publicação de seu caso por meio de sua assinatura em Termo de Consentimento Livre e Esclarecido (TCLE).

\section{Resultados e Discussão}

No caso apresentado, foi indicada a correção da curvatura do sorriso com laminados cerâmicos, os quais permitiram também correções de forma e cromia dentária. Para Suguino et al. (1996), o comprimento esquelético reduzido maxilar pode comprometer a relação entre os incisivos e caninos superiores e o lábio inferior ${ }^{19}$. Segundo Marson et al. (2014), um dos requisitos fundamentais para um sorriso atraente é que os dentes anterossuperiores repousem na linha seco molhada do lábio inferior ${ }^{20}$.

Optou-se pelo escaneamento digital no lugar da moldagem tradicional com one de adição, uma vez que o rompimento do ponto de contato foi necessário em ta Ciência Plural. 2019; 5(1):113-123 
quase todos os elementos dentários. Desta forma, foi planejada uma técnica que oferecesse o mínimo de distorções e máximo de adaptações possíveis, sem a necessidade de troquelização de forma manual. No estudo clínico de Pradies et al. (2015) em que foi avaliado a adaptação interna de coroas cerâmicas pelas técnicas de moldagem com silicone e escaneamento intra oral, foi observado que as adaptações foram significativamente melhores quando realizado por meio do escaneamento digital $^{21}$. Já em estudo laboratorial in vitro, que avaliou gaps marginais em coroas de dissilicato de lítio com moldagem tradicional em silicone e dois tipos de escaneamentos, observaram que os valores médios de gaps foram inferiores nos grupos do escaneamento digital ${ }^{5}$.

A técnica de escaneamento foi rápida, simples, e a paciente não relatou desconforto durante o procedimento. Ademais, na fase de prova dos laminados, o índice de adaptação foi alto, necessitando de pouco ajuste nas peças cerâmicas. Em estudo clínico com 48 pacientes, Gjelvold et al. (2016), avaliaram a técnica de impressão digital (Trios 3Shape) e a técnica de impressão convencional para os seguintes parâmetros: tempo de procedimento, avaliação da dificuldade técnica, desconforto do paciente, adaptação marginal, oclusal e interproximal ${ }^{14}$. A técnica digital, na escala VAS (0 a 100), foi considerada mais fácil (24,00 contra 48,02 no método convencional), menos desconfortável (6,50 e 44,86 para a técnica tradicional) além de apresentar melhores resultados clínicos nas adaptações das peças.

A cerâmica de escolha para a confecção dos laminados foi o dissilicato de lítio, o qual foi realizado de forma injetada e posteriormente maquiada. Esta classe de cerâmica mais moderna permite melhorias na resistência além de ser possível reproduzir com ela cor e translucidez semelhante ao esmalte dentário ${ }^{21}$. Em estudo clínico com 312 pacientes e 800 restaurações em dissilicato de lítio (coroas, facetas, inlays e onlays), foi observado que após 3 anos de acompanhamento, as taxas de obrevivência cumulativa variaram de 95,46\% a 100\%22. Já no estudo clínico, Gehrt et 3. (2013), em que foi avaliado 94 coroas anteriores e posteriores confeccionadas no esmo material, foi observado que após 5 anos, obtiveram uma taxa de sobrevida em \% e após 8 anos, 94,8\%. 
Devido à espessura dos laminados (média de $0,5 \mathrm{~mm}$ ), foi selecionado o cimento resinoso fotoativado para adesão das peças protéticas ao substrato dental. Estes materiais geralmente são utilizados para a cimentação deste tipo de restaurações, uma vez que proporcionam uma estética adequada, baixa solubilidade em ambiente oral, alta força de adesão entre os dentes e as cerâmicas e com propriedades mecânicas adequadas $^{24}$. Em estudo laboratorial Pissaia et al. (2015) avaliou a estabilidade de cor de diferentes cimentos resinosos com tipo de cura diferentes. Os valores de variação de cor $(\Delta \mathrm{E})$ para espécimes duais foram significativamente mais altos do que para os respectivos espécimes fotopolimerizáveis ${ }^{25}$. Desta forma, em situações onde a espessura do laminado cerâmico não vai impedir a passagem de luz para a cura do cimento, deve-se optar por cimentos resinosos fotoativados para melhor estética e estabilidade de $\operatorname{cor}^{25}$.

\section{Conclusão}

A tecnologia empregada se mostrou eficiente na resolução do caso apresentado, sendo esta, uma técnica rápida, que causou pouco desconforto à paciente e oportunizou uma boa adaptação dos laminados cerâmicos.

\section{Referências}

1. Mokhtar HÁ, Abuljadayel LW, Al-Ali RM, Yousef M. The perception of smile attractiveness among Saudi population. Clinical, cosmetic and investigational dentistry. 2015;7(1):17-23.

2. Dawood A, Marti BM, Sauret-Jackson V, Darwood A. 3D printing in dentistry. British dental jornal. 2015;219(11):521-529.

3. Alghazzawi TF, Advancements in CAD/CAM technology: options for practical implementation. Journal of prosthodontic research. 2016;60(2):72-84.

Rashid R, Ayoub WU. Veneers: Modern Approach of Dentistry-A Review. ternational Journal of Advance Research, Ideas and Innovations in Technology. 017;3(5):107-111.

Pradíes G, Zarauz C, Valverde A, Ferreiroa A, Martínez-Rus F. Clinical luation comparing the fit of all-ceramic crowns obtained from silicone and digital 
intraoral impressions based on wavefront sampling technology. Journal of dentistry. 2015;43(2):201-208.

6. Parajuli PK, George S, Shenoy V. An in-vitro study to evaluate the accuracy of dies obtained from dual arch impression trays using addition silicone impression material and tray combinations. Health Renaissance. 2015;12(2):111-117.

7. Bósio JÁ, Del Santo M, Jacob H, Helder B. Odontologia digital contemporâneascanners intraorais digitais. Ortho Sci., Orthod. sci. Pract. 2017;10(39):355-362.

8. Dartora G, Dartora N, Radaelli MB, Alessandretti R, Spazzin AO, Bacchi A. Precisão dos sistemas CAD/CAM em restaurações unitárias: revisão de literatura. Prosth. Lab. Sci. 2014;3(10):133-139.

9. Bernardes SR. Tecnologia CAD/CAM aplicada a prótese dentária e sobre implantes. Jornal ILAPEO. 2012;6(1):8-13.

10. Soares PV, Filho PCFS, Menezes MS, Simamoto VRN, Quagliatto OS, Soares CJ, Raposo LHA, Neves FD. Facetas cerâmicas minimamente invasivas lentes de contato: fundamentos e protocolos. Pro-odonto prótese e dentística. 2015;6(2):9-46.

11. Silva FCFA, Souza LC, Rodrigues NS, Cunha DA, Apolonio FM, Saboia VPA. Técnica de moldagem modificada usando silicona de adição. Revista da Associacao Paulista de Cirurgioes Dentistas. 2016;70(4):364-368.

12. Abdel-Azim T, Rogers K, Elathamna E, Zandinejad A, Metz M, Morton D. Comparison of the marginal fit of lithium disilicate crowns fabricated with CAD/CAM technology by using conventional impressions and two intraoral digital scanners. Journal of Prosthetic Dentistry. 2015;114(4):554-559.

13. Wesemann C, Muallah J, Mah J, Axel B. Accuracy and efficiency of full-arch digitalization and 3D printing: A comparison between desktop model scanners, an intraoral scanner, a CBCT model scan, and stereolithographic 3D printing. Quintessence International. 2017;48(1):41-50.

14. Gjelvold B, Chrcanovic BR, Korduner EK, Collin-Bagewitz I. Intraoral digital impression technique compared to conventional impression technique. A randomized clinical trial. Journal of Prosthodontics. 2016;25(4):282-287.

15. Jain R, Supriya BS, Gupta K. Recent Trends of 3-D Printing in Dentistry-A review. Ann Prosthodont Rest Dent. 2016;2(1):101-104.

16. Serag M, Nassar TA, Avondoglio D, Weiner S. A comparative study of the accuracy of dies made from digital intraoral scanning vs. elastic impressions: An in vitro study. Journal of Prosthodontics. 2018;27(1):88-93.

17. Patzelt SBM, Emmanouilidi A, Stampf S, Strub JR, Att W. Accuracy of full-arch cans using intraoral scanners. Clinical oral investigations. 2014;18(6):1687-1694.

Rhee YK, Huh YH, Cho LR, Park CJ. Comparison of intraoral scanning and nventional impression techniques using 3-dimensional superimposition. The urnal of advanced prosthodontics. 2015;7(6):460-467.

Suguino R, Ramos AL, Terada HH, Furquim LZ, Maeda L, Filho OGS. Análise al. Rev Dental Press Ortod Ortop Maxilar. 1996;1(1):86-107.

ta Ciência Plural. 2019; 5(1):113-123 
20. Marson FC, Piloto RL, Rocha OO, Lolli LF, Progiante PS, Silva CO. Percepção da atratividade do sorriso. Revista UNINGÁ Review. 2014;20(1):26-29.

21. Batson ER, Cooper LF, Duqum I, Mendonça G. Clinical outcomes of three different crown systems with CAD/CAM Technology. J. prosthet. dent. 2014;112(4):770-777.

22. Fabbri G, Zarone F, Dellificorelli G, Cannistraro G, De Lorenzi M, Mosca A, Sorrentino R. Clinical evaluation of 860 anterior and posterior lithium disilicate restorations: retrospective study with a mean follow-up of 3 years and a maximum observational period of 6 years. International Journal of Periodontics \& Restorative Dentistry. 2014;34(2):164-177.

23. Gehrt M, Wolfart S, Rafai N, Reich S, Edelhoff D. Clinical results of lithiumdisilicate crowns after up to 9 years of service. Clin. Oral Invest. 2013;17(1):275-284.

24. Cho SH, Lopez A, Berzins DW, Prasad S, Ahn KW. Effect of different thicknesses of pressable ceramic veneers on polymerization of light-cured and dualcured resin cements. The journal of contemporary dental practice. 2015;16(5):347-352.

25. Pissaia JF, Correr GM, Gonzaga CC, Cunha LF. Influence of shade, curing mode, and aging on the color stability of resin cements. Brazilian Journal of Oral Sciences. 2015;14(4):272-275.

Submetido: $10 / 01 / 2019$

Aprovado: 30/04/2019 